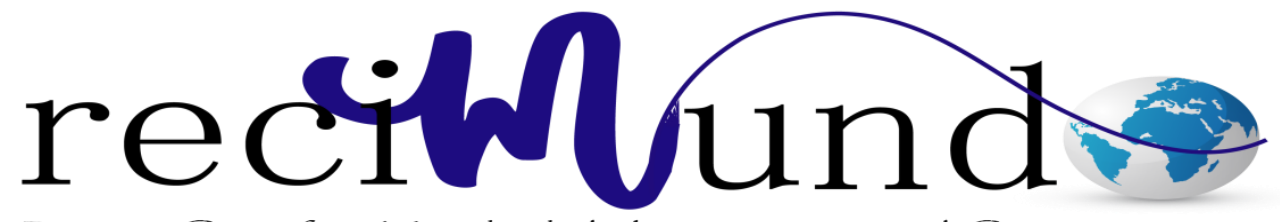

Revista Científica Mundo de la Investigación y el Conocimiento

Luisa María Vega Benavides ${ }^{\text {a }}$; Christian Eduardo Almendáriz Rodríguez ${ }^{\text {; }}$ Iván Arturo Navarro Veliz ${ }^{\mathrm{c}}$; Danny Xavier Almendáriz Rodríguez ${ }^{\mathrm{d}}$

Elaboración de un plan para construir la cimentación de un edificio de tres plantas

Revista Científica Mundo de la Investigación y el Conocimiento. Vol. 2 núm.2, mayo, ISSN: 2588-073X, 2018, pp. 326-355

DOI: 10.26820/recimundo/2.(2).2018.326-355

Editorial Saberes del Conocimiento

Recibido: 05/12/2017 Aceptado: $15 / 02 / 2018$
a. Universidad de Guayaquil.
b. Universidad de Guayaquil.
c. Universidad de Guayaquil.
d. Universidad de Guayaquil. 


\section{Elaboración de un plan para construir la cimentación de un edificio de tres \\ plantas}

Vol. 2, núm. 2., (2018)

Luisa María Vega Benavides; Christian Eduardo Almendáriz Rodríguez; Iván Arturo Navarro Veliz; Danny Xavier Almendáriz Rodríguez

\section{RESUMEN}

La elaboración de un plan constructivo de una cimentación, el cual su enfoque principal es explicar detalladamente el proceso constructivo de esta infra-estructura que es la encargada de soportar y trasmitir la carga de la súper estructura al suelo, la cual va estar apoyada sobre los estratos de suelo ya mejorados. Esta obra consiste en construir una cimentación la cual ya se le han efectuado los estudios pertinentes como es la información geotécnica de los estudios de suelo, con la cual se obtuvo el diseño adecuado de cimentación. Por lo cual se realizaron análisis minucioso de precios unitarios tomando en consideración mano de obra -cuadrilla-, maquinaria y materiales a utilizar. Para esto se consideró también la experiencia en campo de ingenieros, sobre el rendimiento de las cuadrillas según el rubro a utilizar. Ya que como factor principal depende de los rendimientos, el precio unitario de cada rubro. En base a los rendimientos se podrán ejecutar actividades dándole así tiempo de inicio y fin a cada actividad. Los trabajos a ejecutarse los veremos reflejados en un crono grama valorado, el cual nos indicara su inicio y fin, por rubro en cantidad de días, que según los rendimientos, se prolongara su duración.

Palabras clave: Construcción; cimentación; edificios altos 3 plantas; km 9 1/2 vía Daule. 


\title{
Elaboración de un plan para construir la cimentación de un edificio de tres plantas
}

Vol. 2, núm. 2., (2018)

Luisa María Vega Benavides; Christian Eduardo Almendáriz Rodríguez; Iván Arturo Navarro Veliz; Danny Xavier Almendáriz Rodríguez

\begin{abstract}
The elaboration of a constructive plan of a foundation, whose main focus is to explain in detail the constructive process of this infra-structure that is in charge of supporting and transmitting the load of the super structure to the ground, which will be supported on the soil layers already improved. This work consists of building a foundation which has already been carried out the relevant studies such as the geotechnical information of the soil studies, with which the proper foundation design was obtained. For this reason, a detailed analysis of unit prices was carried out, taking into consideration the workforce, the machinery, and the materials to be used. For this, the experience in the field of engineers was also considered, on the performance of the crews according to the item to be used. Since the main factor depends on the yields, the unit price of each item. Based on the yields, activities can be executed, giving start and end time to each activity. The works to be executed will be reflected in a valued time schedule, which will indicate its beginning and end, by item in number of days, which according to the yields, will last its duration.
\end{abstract}

Key words: Building; foundation tall buildings 3 floors; km 9 1/2 via Daule. 


\section{Elaboración de un plan para construir la cimentación de un edificio de tres \\ plantas}

Vol. 2, núm. 2., (2018)

Luisa María Vega Benavides; Christian Eduardo Almendáriz Rodríguez; Iván Arturo Navarro Veliz; Danny Xavier Almendáriz Rodríguez

\section{Introducción.}

La elaboración de un plan constructivo de una cimentación, el cual su enfoque principal es explicar detalladamente el proceso constructivo de esta infra-estructura que es la encargada de soportar y trasmitir la carga de la súper estructura al suelo, la cual va estar apoyada sobre los estratos de suelo ya mejorados. (Toirac Corral, 2008) (Rodolfo, 1991)

Al hablar de cimentación nos estamos refiriendo a la parte más importante en lo que comprende una estructura ya que esta debe estar bien diseñada para soportar grandes cargas que van a ser apoyadas sobre ella y a su vez van a ser trasmitidas al suelo el cual debe tener un buen mejoramiento, si así lo requiere, para que no se efectúen grandes asentamientos los cuales ocasionarían daños a la súper estructura. (Britez, Gadea, \& Carvalho, 2016) (Associação Brasileira de Normas Técnicas, 2006) (Spenser, 2014)

Como requisito principal para realizar un diseño de cimentación es indispensable contar con información geotécnica y el tipo de estructura de diseño superficial.

Esta obra consiste en construir una cimentación la cual ya se le han efectuado los estudios pertinentes como es la información geotécnica de los estudios de suelo, con la cual se obtuvo el diseño adecuado de cimentación.

El objetivo de la presente investigación es examinar minuciosamente los pasos a seguir en el plan constructivo, partiendo desde el estudio de planos y resultados de pruebas de laboratorio, para la cimentación de un edificio de tres plantas. 


\section{Elaboración de un plan para construir la cimentación de un edificio de tres plantas}

Vol. 2, núm. 2., (2018)

Luisa María Vega Benavides; Christian Eduardo Almendáriz Rodríguez; Iván Arturo Navarro

Veliz; Danny Xavier Almendáriz Rodríguez

\section{Metodología Constructiva}

\section{Preliminares}

Desbroce, limpieza y desalojo del terreno

Se realiza la limpieza del terreno retirando toda el área verde que se encuentre en el sitio.

Se procede a usar maquinaria la cual va a raspar el terreno para proceder a trabajar de mejor manera.

Se inició el trabajo en la obra haciendo la limpieza y desbroce del terreno, trabajando con maquinaria de las siguientes características:

Maquinaria - retroexcavadora.

- Peso - 6.7 toneladas.

- Potencia- 65HP.

- Capacidad - 0.77 metros cúbicos.

\section{Volqueta mula}

- $12 \mathrm{~m}^{3}$

Con la cual se procede al desalojo de material, que se lo desecha en espacios oficialmente decretados. También se efectúan desbroce de vegetación que se trasladan hacia áreas de buena condición. 


\section{Elaboración de un plan para construir la cimentación de un edificio de tres}

plantas

Vol. 2, núm. 2., (2018)

Luisa María Vega Benavides; Christian Eduardo Almendáriz Rodríguez; Iván Arturo Navarro Veliz; Danny Xavier Almendáriz Rodríguez

\section{Figura $N^{\circ}$ 1.- Corte de terreno usando maquinaria}

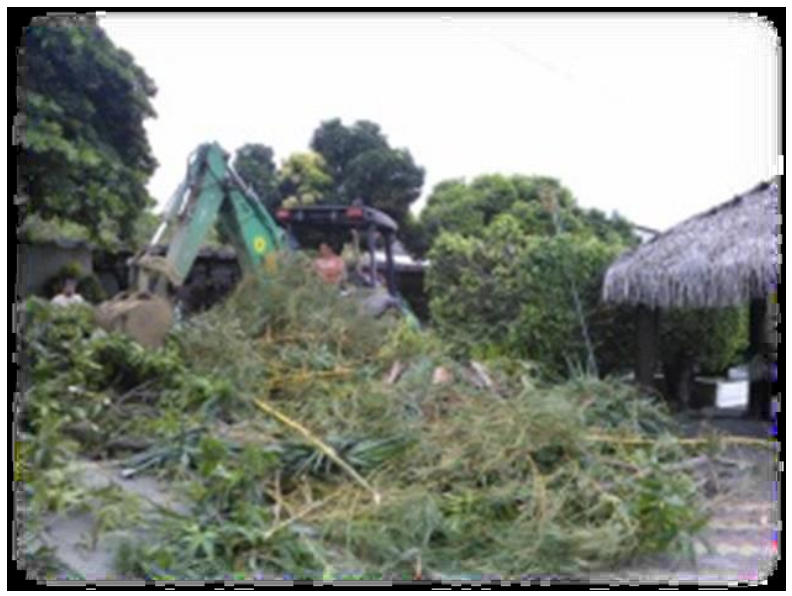

Referencia: Fuente Propia

Figura $N^{\circ}$ 2.- Desalojo de Material

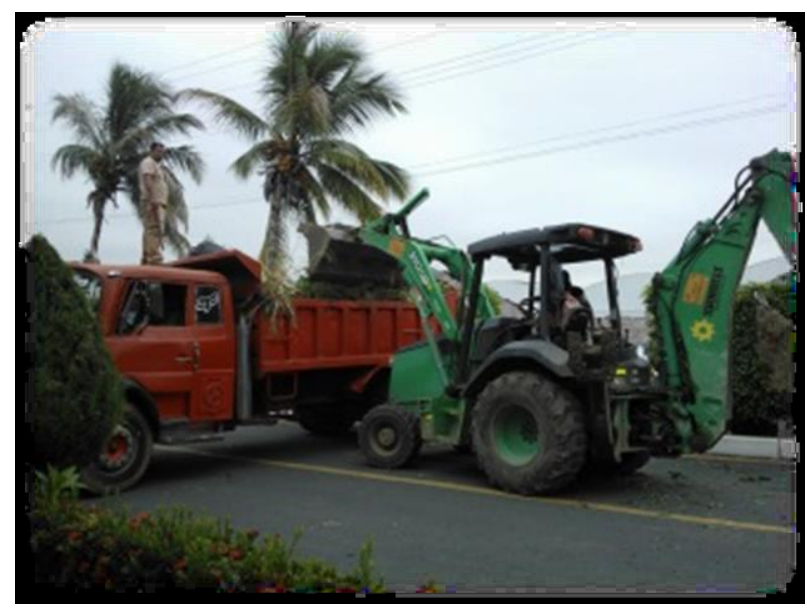

Referencia: Fuente Propia 


\section{Elaboración de un plan para construir la cimentación de un edificio de tres plantas}

Vol. 2, núm. 2., (2018)

Luisa María Vega Benavides; Christian Eduardo Almendáriz Rodríguez; Iván Arturo Navarro Veliz; Danny Xavier Almendáriz Rodríguez

El área total de limpieza y desbroce es de 42 m largo y 15 m de ancho, el cual se realizó con maquinaria.

\section{Cerramiento Perimetral-Lona}

Se dará un margen del terreno, el cual va estar limitado por una lona que indicara que toda esa área va ser utilizada.

Se ubicara de forma lineal en todo el perímetro del área a usar, esto quiere decir que será el margen del terreno.

El cual se utilizaran cuartones de madera de $2 \mathrm{~m}$ de altura cada $4 \mathrm{~m}$ de distancia haciendo hueco de $30 \mathrm{~cm}$ de ancho por $30 \mathrm{~cm}$ de profundidad para darle estabilidad al cuartón que va a ser de soporte para la lona, luego que se encuentren bien ubicados los cuartones se procede a colocar la lona en todo el perímetro ya establecido en el terreno.

\section{Caseta de Guardián - Bodega}

Se realiza construcción de las áreas de caseta de guardián y bodega para almacenar los materiales de construcción.

Estas áreas son construidas provisionalmente y su ejecución se da antes de iniciar propiamente la obra.

Luego de establecer las dimensiones de la caseta de guardián - bodega que son $6.5 \mathrm{~m}$ de longitud x 5.6m de ancho. 


\section{Elaboración de un plan para construir la cimentación de un edificio de tres \\ plantas}

Vol. 2, núm. 2., (2018)

Luisa María Vega Benavides; Christian Eduardo Almendáriz Rodríguez; Iván Arturo Navarro Veliz; Danny Xavier Almendáriz Rodríguez

Para así iniciar la construcción de la misma lo primero a realizar son los orificios con dimensiones de $0.3 \mathrm{~m}$ de ancho $0.3 \mathrm{~m}$ de largo y 0.4 de alto para la colocación de cuartones que van a ser utilizados como columnas.

Los cuartones a utilizar tendrán las siguientes dimensiones de altura, los que se encuentran ubicados en la parte principal serán de $2.75 \mathrm{~m}$ y los que se encuentran en la parte secundaria tendrán $3 \mathrm{~m}$.

Estos cuartones que van a trabajar como columnas se las realizara usando nivel de mano aplomadas.

Se procederá a colocar cuartones en la parte inferíos, media y superior los cuales trabajaran como vigas.

Finalmente se cierra el cuadrante de caseta de guardianía bodega usando tablas de madera semidura para la colocación de paredes con una altura de $3 \mathrm{~m}$. Y se procede a colocar la cubierta, la cual va estar conformada de zinc, la misma que va estar clavada a los cuartones transversales, los cuales van a estar ubicados a una separación de $1.50 \mathrm{~m}$ de distancia el uno del otro.

\section{Instalación Provisional Eléctrica}

Se instalaran puntos eléctricos donde se utilizara tubo, cableado y demás accesorias para suministrar energía a las diferentes áreas provisionales que así lo requiera.

Estos puntos serán ubicados dentro de la oficina, caseta de guardián y bodega los cuales serán utilizados para suministrar energía a los aparatos electrónicos como son computadoras, 


\section{Elaboración de un plan para construir la cimentación de un edificio de tres plantas}

Vol. 2, núm. 2., (2018)

Luisa María Vega Benavides; Christian Eduardo Almendáriz Rodríguez; Iván Arturo Navarro Veliz; Danny Xavier Almendáriz Rodríguez

impresoras y demás objetos que se encuentren en estos lugares. También de estos puntos eléctricos provisionales se suministrara energía para los equipos utilizados en obra que así lo requieran.

Esta instalación será únicamente provisional esto quiere decir que su uso será solo durante el proceso constructivo de dicha obra, ya que al momento de entregar la obra estos puntos se retiraran.

Trazado y Replanteo

Este trabajo se realiza para ubicación exacta en planta y en nivel los cimientos que serán construidos. Para esto se utilizara dimensiones reales que se encuentran en los planos suministrados empleando cal se delimitara el terreno a usar.

Para esto se realiza un trabajo topográfico en campo, donde el contratista determina la ubicación exacta en planta y en nivel de las obras por construir, de acuerdo con los planos suministrados. Se tiene que trazar exactamente lo planteado en el plano hacia el terreno. Se dibuja el área donde va ubicada la cimentación que se encuentran marcadas en los planos. El Contratista ejecutará el trazado, replanteo y nivelación de la construcción en planta y nivel, utilizando para ello todos los instrumentos de precisión que fuesen necesarios, empleando los servicios de un topógrafo.

Se procederá a ubicar los ejes, a los cuales serán referenciados con el uso de caballetes o vallas instalándolos en el área del terreno de construcción. 


\section{Elaboración de un plan para construir la cimentación de un edificio de tres}

plantas

Vol. 2, núm. 2., (2018)

Luisa María Vega Benavides; Christian Eduardo Almendáriz Rodríguez; Iván Arturo Navarro Veliz; Danny Xavier Almendáriz Rodríguez

\section{Figura $N^{\circ}$ 3.- Caballete}

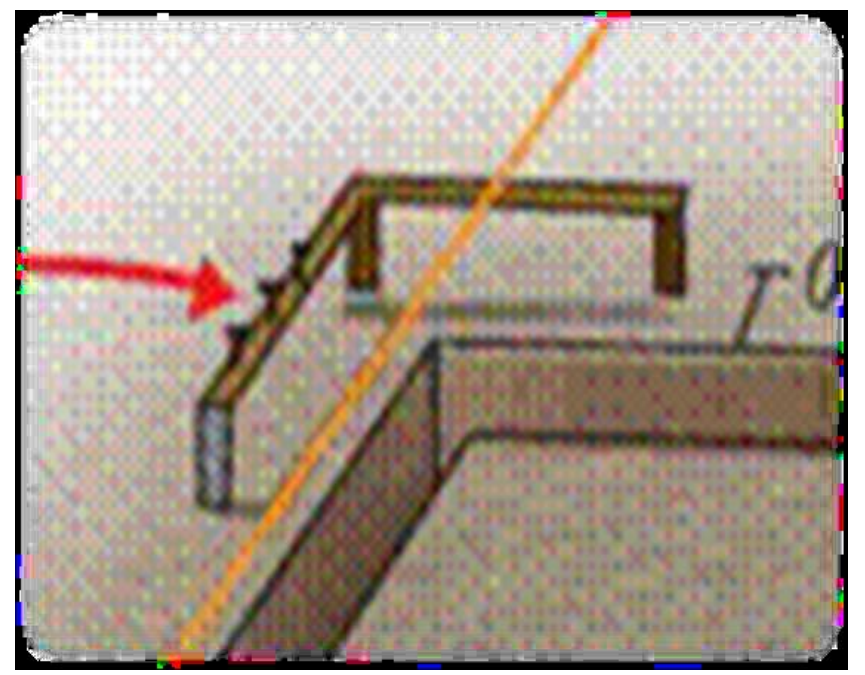

Referencia: construccion.org.

El proceso de este trabajo se realiza penetrando sobre el terreno cuartones o estacas de dimensiones $1.5 \mathrm{~m} \mathrm{x} \mathrm{2"} 2^{\prime \prime}$, las cuales van a ser sostenidas por una tabla de madera semiduras, con una distancia de $3 \mathrm{~m}$ con un ancho de $0.20 \mathrm{~m}$ y $0.02 \mathrm{~m}$ de espesor. Las mismas que van estar a una distancia de $1.20 \mathrm{~m}$ de la superficie del terreno.

Para anclar las estacas con las tablas de madera semidura se requiere del uso de clavos de 2“x 8. Los cuales también nos permitirán usarlos como referencia siendo así los ejes que van estar delimitados por una piola la cual va estar intersectada de esquina a esquina. La ubicación de los caballetes va estar alejado de los ejes de edificación, para así poder mantener su instalación original sin que sean removidos por molestias en el proceso constructivo. 


\section{Elaboración de un plan para construir la cimentación de un edificio de tres plantas}

Vol. 2, núm. 2., (2018)

Luisa María Vega Benavides; Christian Eduardo Almendáriz Rodríguez; Iván Arturo Navarro

Veliz; Danny Xavier Almendáriz Rodríguez

Figura $N^{\circ}$ 4.- Realización de trazados y replanteo

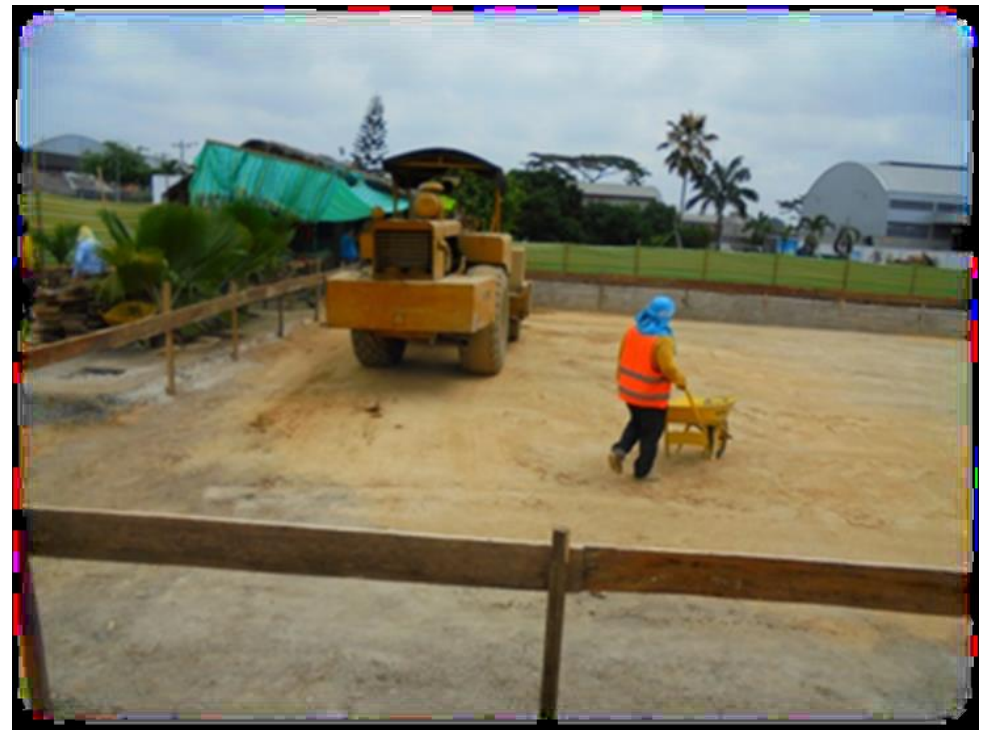

Referencia: Fuente Propia

\section{Movimiento de tierra}

Excavación y desalojo

Se realiza la excavación según lo requiera el sitio tomando en consideración los estudios de suelo. Se excavara el área delimitada por el trazado donde la profundidad será dada por los estudios de suelo.

Para realizar esta excavación se utilizó:

Maquinaria - retroexcavadora

- Peso - 6.7 toneladas. 


\section{Elaboración de un plan para construir la cimentación de un edificio de tres}

plantas

Vol. 2, núm. 2., (2018)

Luisa María Vega Benavides; Christian Eduardo Almendáriz Rodríguez; Iván Arturo Navarro Veliz; Danny Xavier Almendáriz Rodríguez

- Potencia- 65HP.

- Capacidad - 0.77 metros cúbicos.

\section{Volqueta mula}

- $12 \mathrm{~m}^{3}$

Figura $N^{\circ}$ 5.- Excavación en el terreno

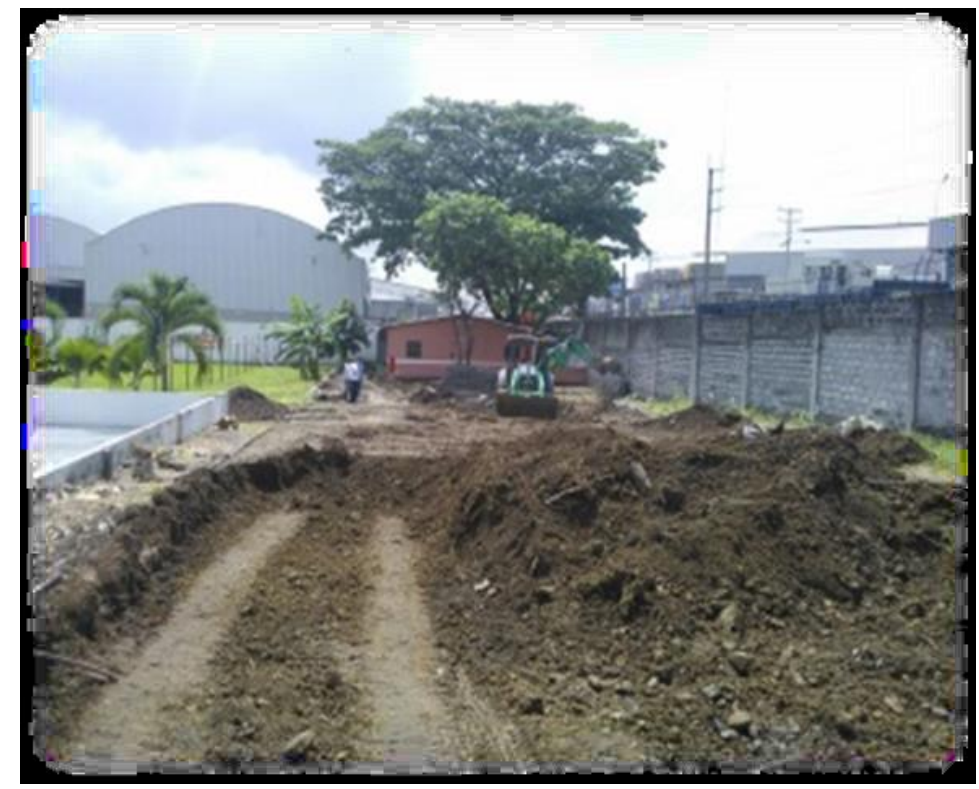

Referencia: Fuente Propia

Se realizó excavación con una profundidad de $20 \mathrm{~cm}$ con un ancho de $14.5 \mathrm{~m}$ y un longitud de $41.5 \mathrm{~m}$.

El material extraído se lo mantiene en la obra para luego ser utilizado dentro de la construcción. 


\section{Elaboración de un plan para construir la cimentación de un edificio de tres plantas}

Vol. 2, núm. 2., (2018)

Luisa María Vega Benavides; Christian Eduardo Almendáriz Rodríguez; Iván Arturo Navarro Veliz; Danny Xavier Almendáriz Rodríguez

\section{Mejoramiento de suelo con material importado}

Con este material importado -cascajo- se procede a rellenar el área que ya fue delimitada para obtener los niveles de cota establecidos que se requieren en el terreno.

Rellenar una primera capa de piedraplen -GW- de 0.40m, para mejorar el terreno, compactada al $100 \%$, se deberá utilizar rodillo liso vibratorio de 18 Ton. La capa en contacto con la cimentación será de espesor $0.90 \mathrm{~m}$, constituida por material de sub-base clase 3 . Esta será de plataforma de contacto suelo-estructura. Considerará que el sub suelo desarrolla una capacidad de carga máxima de $16.6 \mathrm{Tno} / \mathrm{m}^{2}$, se ha considerado factor de seguridad tres.

Figura $N^{\circ}$ 6.- Mejoramiento del terreno con piedra base

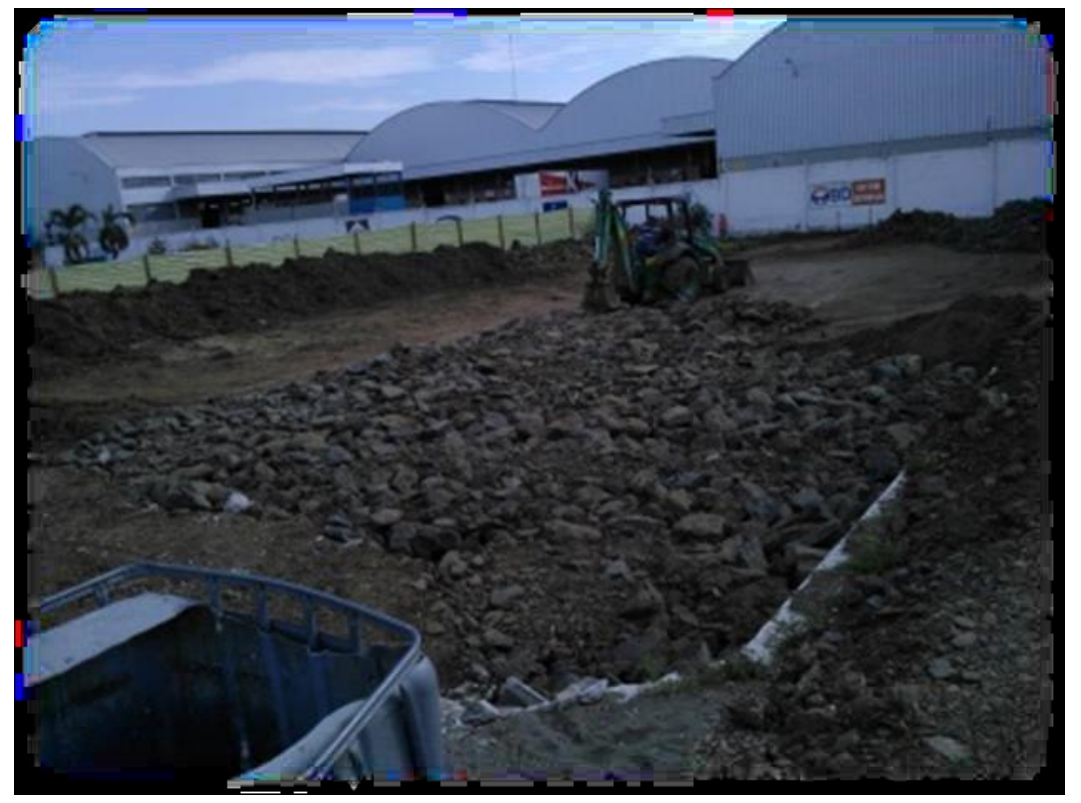

Referencia: Fuente Propia 


\section{Elaboración de un plan para construir la cimentación de un edificio de tres}

plantas

Vol. 2, núm. 2., (2018)

Luisa María Vega Benavides; Christian Eduardo Almendáriz Rodríguez; Iván Arturo Navarro Veliz; Danny Xavier Almendáriz Rodríguez

\section{Figura $N^{\circ}$ 7.- Compactación del terreno con rodillo lizo}

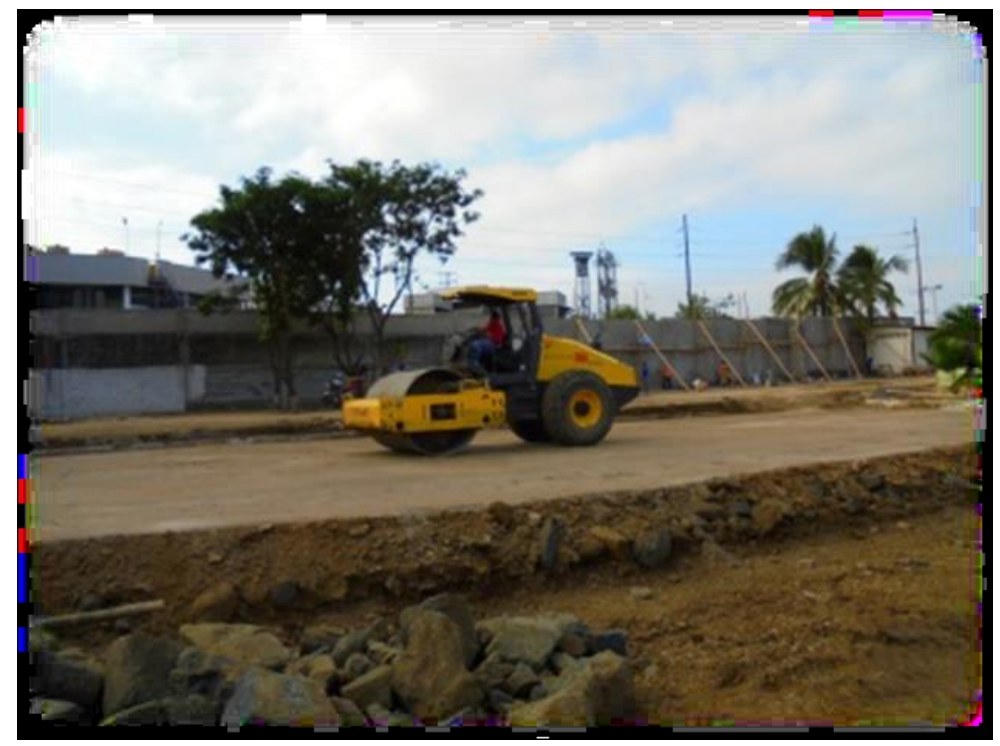

Referencia: Fuente Propia

Antes de realizar la compactación, el material de la capa a compactar, debe tener la humedad óptima. Para que esta compactación cumpla con las especificaciones se verificara si el material a usar necesita ser secado o humedecido ya que este debe tener una humedad uniforme para tener una buena compactación.

El relleno será estrictamente realizado, de acuerdo con lo que indica los estudios de suelo.

El material importado -cascajo- que es depositado al terreno por la volqueta, se lo riega en toda el área requerida a lo largo y a lo ancho hasta obtener una capa máximo de $20 \mathrm{~cm}$ para ser compactada siguiendo las especificaciones. 


\section{Elaboración de un plan para construir la cimentación de un edificio de tres plantas}

Vol. 2, núm. 2., (2018)

Luisa María Vega Benavides; Christian Eduardo Almendáriz Rodríguez; Iván Arturo Navarro

Veliz; Danny Xavier Almendáriz Rodríguez

Posterior mente se procederá a hidratar el material, por medio del uso de tanquero tomando en consideración su velocidad y cantidad de salida del agua.

\section{Relleno manual con material de mejoramiento}

Con este material importado -cascajo mediano- se procede a rellenar el área vacía que se encuentra entre zapatas.

Estos volúmenes varían según las dimensiones de los ejes, este relleno se realiza manualmente ya que la maquinaria no puede entrar a estas áreas reducidas.

\section{Figura $N^{\circ}$ 8.- Relleno Manual}

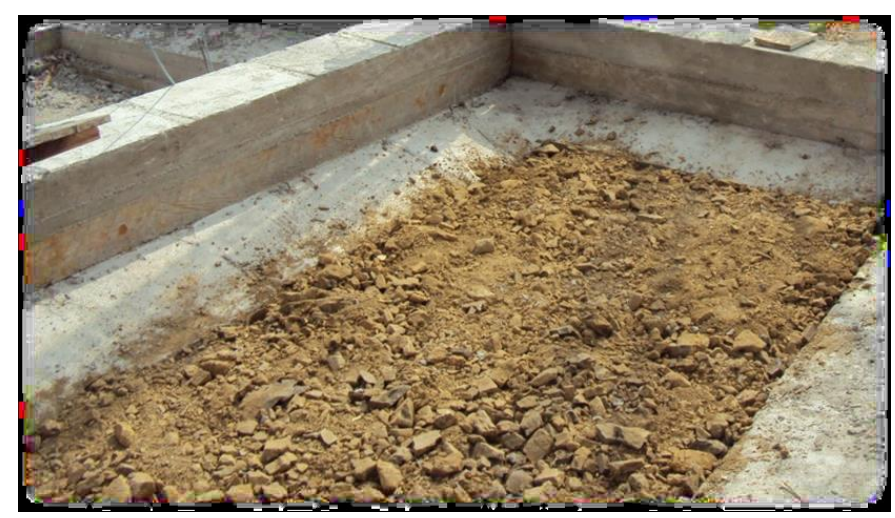

Referencia: Fuente Propia

El relleno se efectuara de manera organizada de tal manera que cada $0.2 \mathrm{~m}$ de relleno se realizara la compactación de dicho material para así tener un suelo totalmente compactado y consolidado, a su vez procurando que este tenga la humedad óptima, llegando a los niveles requeridos por los planos. Para realizar esta compactación se utiliza maquina pequeña la cual es 


\section{Elaboración de un plan para construir la cimentación de un edificio de tres \\ plantas}

Vol. 2, núm. 2., (2018)

Luisa María Vega Benavides; Christian Eduardo Almendáriz Rodríguez; Iván Arturo Navarro Veliz; Danny Xavier Almendáriz Rodríguez

manipulada por un operador de maquinaria liviana, esta máquina actúa haciendo presión en el suelo, originado por su propio peso y su efecto vibratorio.

Para realizar este trabajo se requiere del uso de carretillas las cuales van a ser manipuladas por los oficiales, por medio de ellas se podrá trasladar el material importado cascajo mediano- el cual va ser depositado en los orificios que se originan en las intersecciones de las zapatas, hasta obtener una capa homogénea no máximo a los $0.20 \mathrm{~m}$ como lo indican las especificaciones, a su vez se tomara en cuenta si el material se encuentra seco o húmedo ya que este debe cumplir con una humedad óptima, luego de que se cumpla lo dicho se procederá a la compactación, la cual se realiza con maquinaria liviana hasta verificar que el suelo se encuentre bien compactado y consolidado. De esta manera se realiza el relleno manual hasta llegar al nivel requerido, realizando todos los pasos por cada capa. Este relleno deberá ser debidamente colocado, tomando en cuenta las especificaciones ya planteadas.

\section{Figura $N^{\circ}$ 9.- Compactación del terreno}

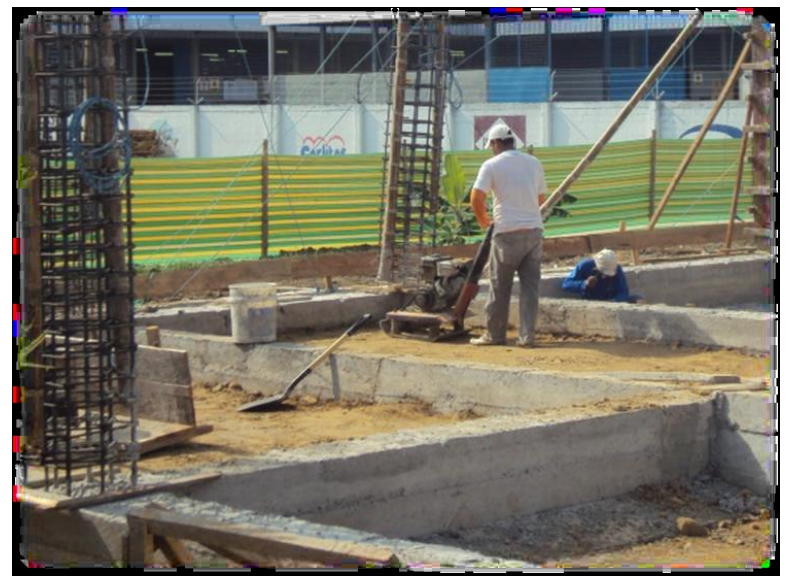

Referencia: Fuente Propia 


\section{Elaboración de un plan para construir la cimentación de un edificio de tres plantas}

Vol. 2, núm. 2., (2018)

Luisa María Vega Benavides; Christian Eduardo Almendáriz Rodríguez; Iván Arturo Navarro Veliz; Danny Xavier Almendáriz Rodríguez

\section{Cimentación}

Replantillo de hormigón f'c140kg/ $\mathrm{cm}^{3}$ e-10 $\mathrm{cm}$

Es una lámina de espesor especificado de hormigón simple que se la coloca entre el suelo y la cimentación. Se debe ubicar en el área donde va ser colocada la zapata el hormigón simple con un espesor de $5 \mathrm{~cm}$. Este debe de mantenerse limpio para la colocación de armadura de la zapata.

Para realizar el replantillo se deberá revisar los agregados los cuales deben cumplir con la dosificación, dada por el ingeniero. Este proceso se lo realizara con el uso de concretera, se deberá mezclar todos los agregados como son:

- Agua.

- Cemento.

- Piedra.

Para lo cual se tomara las cantidades de agregados finos y gruesos por medio del uso de parihuelas.

Para obtener una mezcla de resistencia f'c $140 \mathrm{~kg} / \mathrm{cm}^{3}$ la dosificación medida en parihuelas es:

- $F^{\prime} \mathrm{c} 140 \mathrm{~kg} / \mathrm{cm}^{3}$

- 1 saco de cemento $50 \mathrm{~kg}$

- Arena 3 parihuelas de $15 \mathrm{~cm}$ x $40 \mathrm{~cm}$ x $40 \mathrm{~cm}$ 


\section{Elaboración de un plan para construir la cimentación de un edificio de tres}

plantas

Vol. 2, núm. 2., (2018)

Luisa María Vega Benavides; Christian Eduardo Almendáriz Rodríguez; Iván Arturo Navarro Veliz; Danny Xavier Almendáriz Rodríguez

- Piedra 4 parihuelas de $20 \mathrm{~cm} \times 40 \mathrm{~cm} \times 40 \mathrm{~cm}$

- Agua 170 litros

Figura $N^{\circ}$ 10.- Mezcla del hormigón

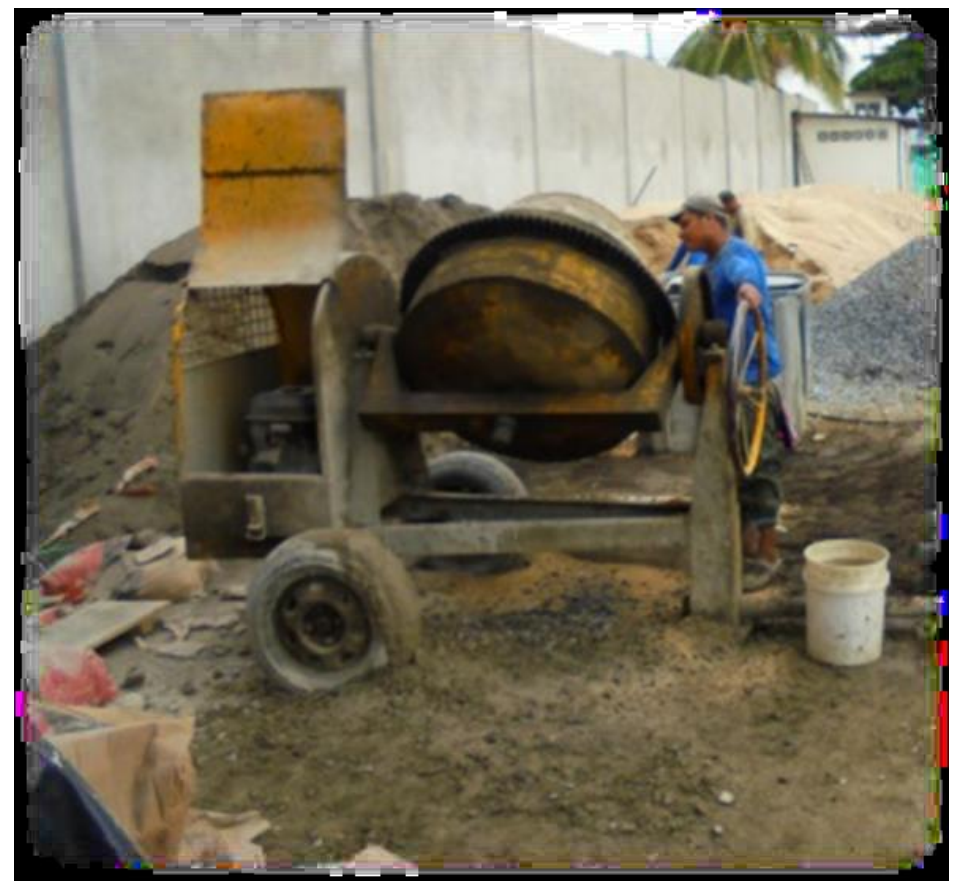

Referencia: Fuente Propia

El agua estará medida en litros, el agua requerida para obtener esta resistencia es de 170litros los cuales corresponden a $0.17 \mathrm{~m}^{3}$.

El tambor de la concretera girara como mínimo 3minutos para poder iniciar con el hormigonado. 


\section{Elaboración de un plan para construir la cimentación de un edificio de tres plantas}

Vol. 2, núm. 2., (2018)

Luisa María Vega Benavides; Christian Eduardo Almendáriz Rodríguez; Iván Arturo Navarro Veliz; Danny Xavier Almendáriz Rodríguez

Esta mezcla se le dará el uso inmediato luego del tiempo establecido ya que no se podrá mantener el concreto agregando más agua porque perderá su dosificación indicada y a su vez perderá resistencia.

Este elemento debe utilizarse de 25 a 30 minutos máximo después de su mezcla, antes de su fraguado inicial. El concreto debe ser aplicado de manera correcta evitando que sus agregados se segreguen, esto quiere decir que se separen los agregados finos de los gruesos, lo ideal sería que el concreto trabaje de manera uniforme. Esta mezcla es transportada por medio de carretillas, que tomara un tiempo de 2 minutos de cargada del hormigón a la carretilla y 2 minutos de vaciado del hormigón al terreno. El terreno donde se vaciara el hormigón debe mantenerse humedecido para que sea de ayuda al momento del fraguado, esto evitara un poco la perdiga de líquido.

Figura $N^{\circ}$ 11.- Replantillo

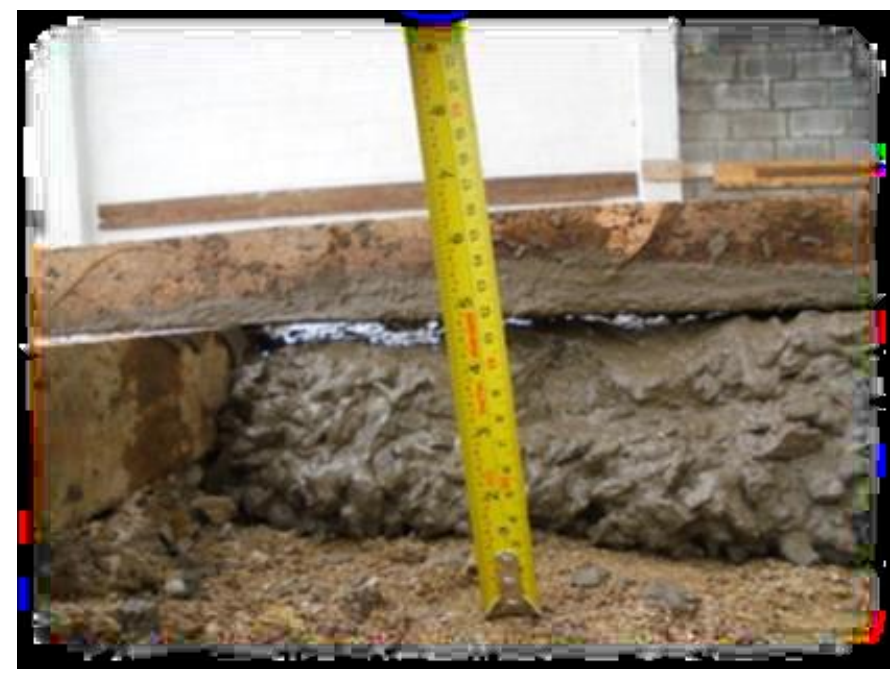




\section{Elaboración de un plan para construir la cimentación de un edificio de tres}

plantas

Vol. 2, núm. 2., (2018)

Luisa María Vega Benavides; Christian Eduardo Almendáriz Rodríguez; Iván Arturo Navarro Veliz; Danny Xavier Almendáriz Rodríguez

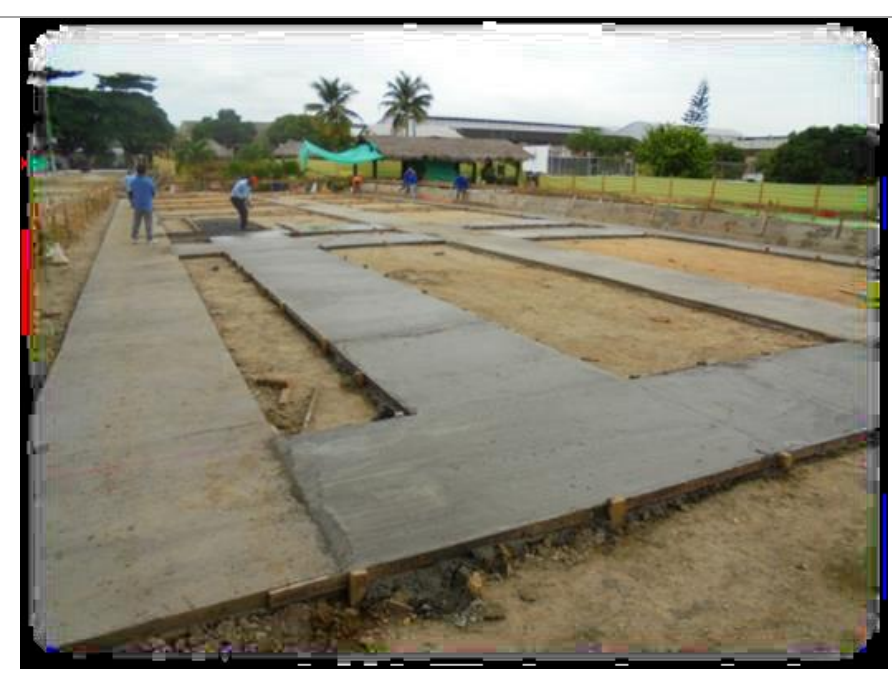

Referencia: Fuente Propia

Acero de refuerzo en barras fy $4200 \mathrm{~kg} / \mathrm{cm} 2$

El acero a colocarse será doblado y cortado según lo especifiquen los planos estructurales.

Al realizar el doblado del acero se debe verificar que no se encuentren fisuras. 


\section{Elaboración de un plan para construir la cimentación de un edificio de tres plantas}

Vol. 2, núm. 2., (2018)

Luisa María Vega Benavides; Christian Eduardo Almendáriz Rodríguez; Iván Arturo Navarro

Veliz; Danny Xavier Almendáriz Rodríguez

Figura $N^{\circ}$ 13.- Procedimiento de doblado

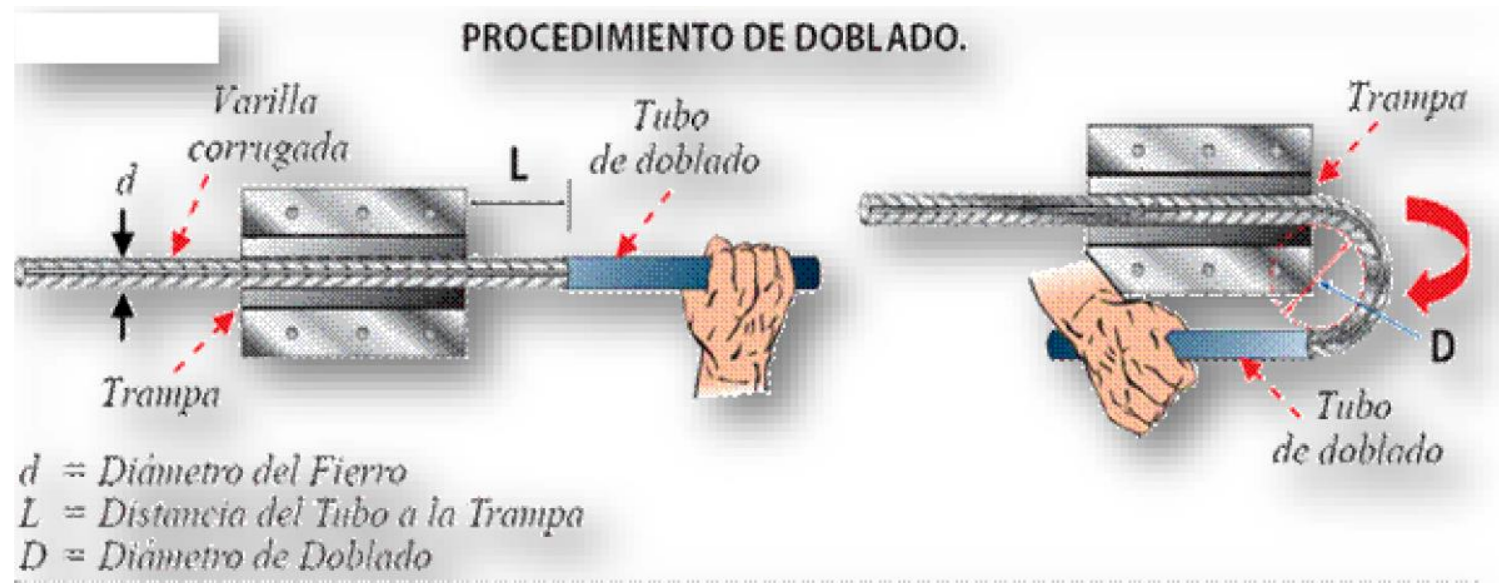

Se debe considerar las dimensiones mínimas que debe tener la varilla.

Figura $N^{\circ}$ 14.- Procedimiento para doblar Bastones

PARA DOBLAR BASTONES

\begin{tabular}{|c|c|c|c|c|}
\hline \multirow[b]{2}{*}{$\begin{array}{l}\text { Diametro } \\
\text { (d) }\end{array}$} & \multicolumn{2}{|c|}{ Distancia (L) } & \multirow{2}{*}{$\begin{array}{l}\text { Dismetro } \\
\text { minimo de } \\
\text { doblado (D) }\end{array}$} & \multirow{2}{*}{$\begin{array}{l}\text { Largo } \\
\text { minimo del } \\
\text { extremo } \\
\text { doblado }\end{array}$} \\
\hline & $\begin{array}{c}\text { Doblez } \\
\text { a } 90^{\circ}\end{array}$ & $\begin{array}{l}\text { Doblex } \\
\text { a } 180^{\circ}\end{array}$ & & \\
\hline $6 \mathrm{~mm}$ & $25 \mathrm{~mm}$ & $55 \mathrm{~mm}$ & $36 \mathrm{~mm}$ & $80 \mathrm{~mm}$ \\
\hline $8 \mathrm{~mm}$ & $30 \mathrm{~mm}$ & $70 \mathrm{~mm}$ & $48 \mathrm{~mm}$ & $100 \mathrm{~mm}$ \\
\hline $3 / 8^{2}$ & $35 \mathrm{~mm}$ & $85 \mathrm{~mm}$ & $57 \mathrm{~mm}$ & $120 \mathrm{~mm}$ \\
\hline $12 \mathrm{~mm}$ & $\$ 0 \mathrm{~mm}$ & $110 \mathrm{~mm}$ & $72 \mathrm{~mm}$ & $140 \mathrm{~mm}$ \\
\hline $1 / 2^{s}$ & $55 \mathrm{~mm}$ & $120 \mathrm{~mm}$ & $76 \mathrm{~min}$ & $150 \mathrm{mim}$ \\
\hline $5 / 8^{2}$ & $65 \mathrm{~mm}$ & $150 \mathrm{~mm}$ & $95 \mathrm{~mm}$ & $190 \mathrm{~mm}$ \\
\hline $3 / 4^{\circ}$ & $85 \mathrm{~mm}$ & $175 \mathrm{~mm}$ & $114 \mathrm{~mm}$ & $230 \mathrm{~mm}$ \\
\hline $1 "$ & $115 \mathrm{~mm}$ & $235 \mathrm{~mm}$ & $152 \mathrm{~mm}$ & $300 \mathrm{~mm}$ \\
\hline
\end{tabular}
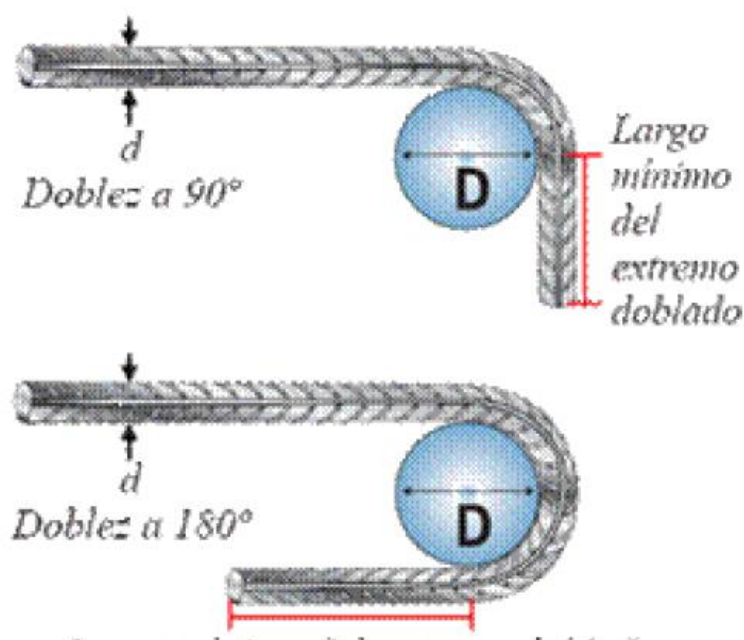

Lango minimo del extremo doblado 


\section{Elaboración de un plan para construir la cimentación de un edificio de tres plantas}

Vol. 2, núm. 2., (2018)

Luisa María Vega Benavides; Christian Eduardo Almendáriz Rodríguez; Iván Arturo Navarro Veliz; Danny Xavier Almendáriz Rodríguez

Figura $N^{\circ}$ 15.- Colocación del acero
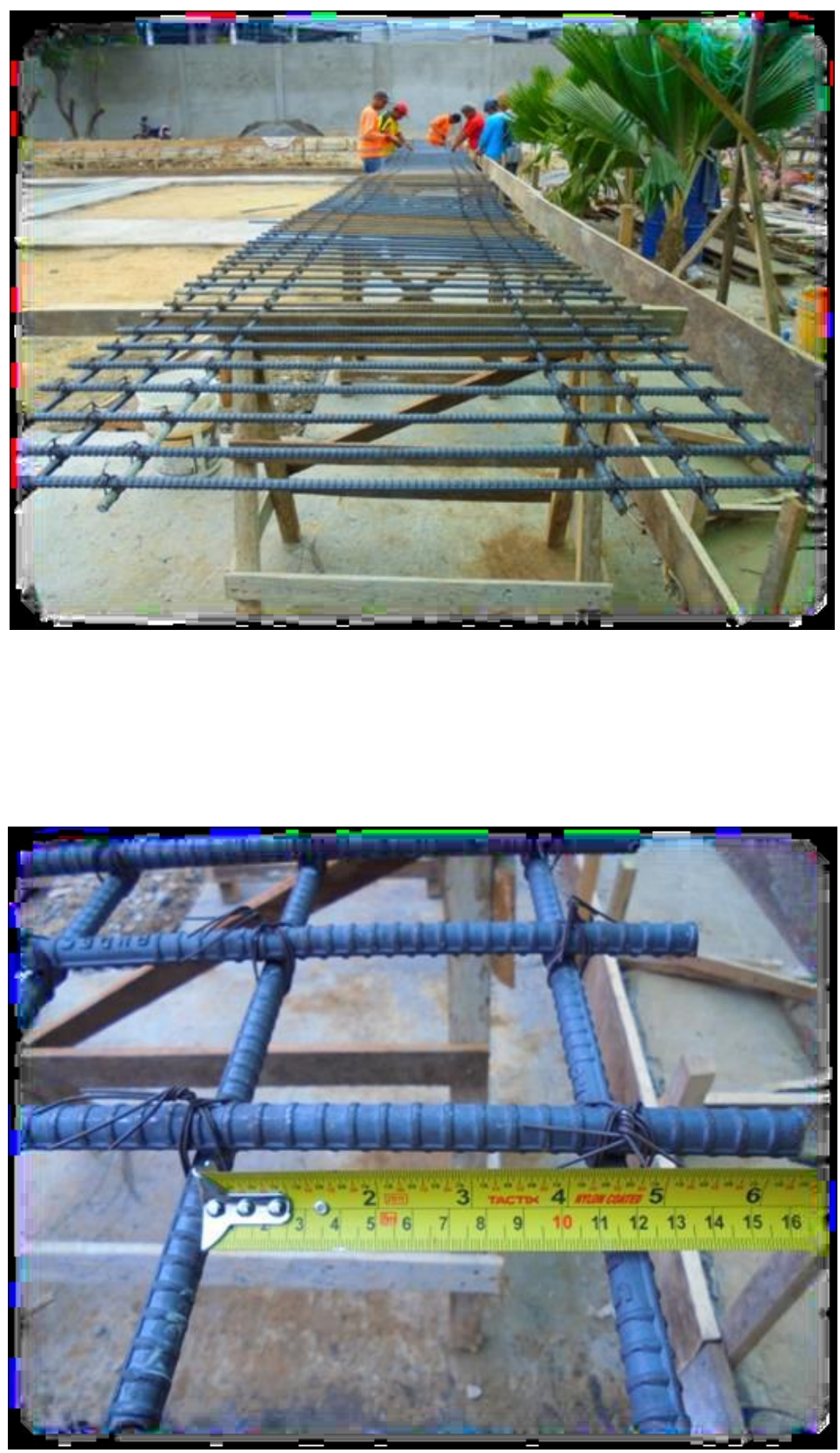


\section{Elaboración de un plan para construir la cimentación de un edificio de tres plantas}

Vol. 2, núm. 2., (2018)

Luisa María Vega Benavides; Christian Eduardo Almendáriz Rodríguez; Iván Arturo Navarro

Veliz; Danny Xavier Almendáriz Rodríguez

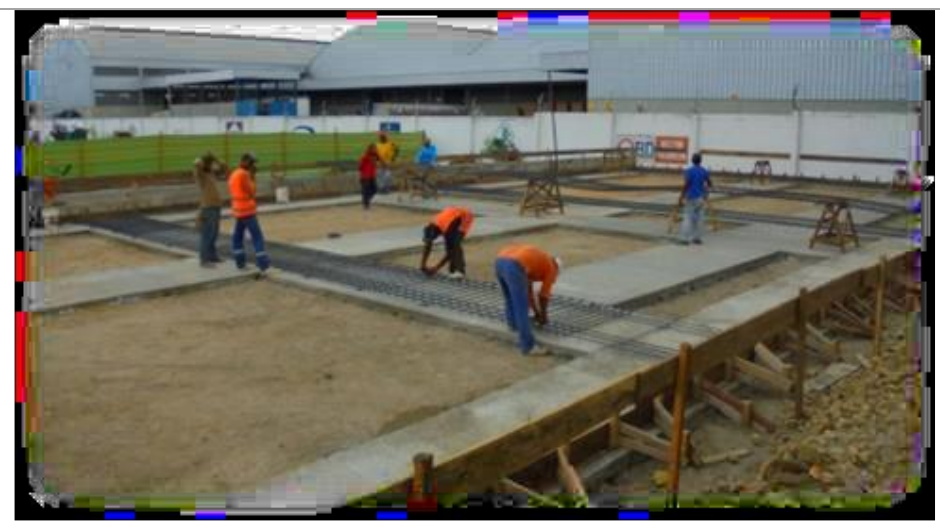

Referencia: Fuente Propia

Zapatas, vigas de amarre y riostras de hormigón $f^{\prime} \mathrm{c}=280 \mathrm{~kg} / \mathrm{cm} 2$.

Construcción de zapata corrida en dos direcciones siguiendo los detalles establecidos en los planos estructurales como son dimensiones.

Se considera las especificaciones sobre el concreto de diseño de f'c $280 \mathrm{~kg} / \mathrm{cm}^{3}$.

Para la realización de la zapata corrida en dos direcciones se debe construir el encofrado, el cual debe ser resistente para soportar el empuje del hormigón, este debe ser construido en base a las dimensiones establecidas en los planos estructurales. El encofrado debe mantenerse limpio sin ningún elemento extraño en su interior, antes de colocar el hormigón se debe humedecer el encofrado para así no permitir la adherencia del concreto con el encofrado y luego poder retirarlo sin dañar la infraestructura. Se debe revisar si el encofrado está colocado de manera correcta verificando el recubrimiento si se encuentra en sus dimensiones establecidas. El encofrado podrá ser reutilizado siempre y cuando este no se encuentre deformado y su superficie tiene que estar libre de restos de hormigón. El encofrado se lo realiza usando tablas, cuartones y clavos, los 


\section{Elaboración de un plan para construir la cimentación de un edificio de tres \\ plantas}

Vol. 2, núm. 2., (2018)

Luisa María Vega Benavides; Christian Eduardo Almendáriz Rodríguez; Iván Arturo Navarro Veliz; Danny Xavier Almendáriz Rodríguez

cuales se los emplea ubicándolos a los costados de la armadura de cimentación ya que este es el encargado de darle la forma al hormigón. Se colocan tablas a lo largo de la zapata usando las medidas indicadas en los planos de la cimentación, las cuales van a ser sustentadas por cuartones que se encontraran fijos al suelo por medio del uso de clavos, que contaran con una dimensión de $0.50 \mathrm{~m}$ de largo y existirá una distancia entre ellos de $0.50 \mathrm{~m}$. el encofrado para las riostras es ubicado de igual manera.

Figura $N^{\circ}$ 16.- Encofrado

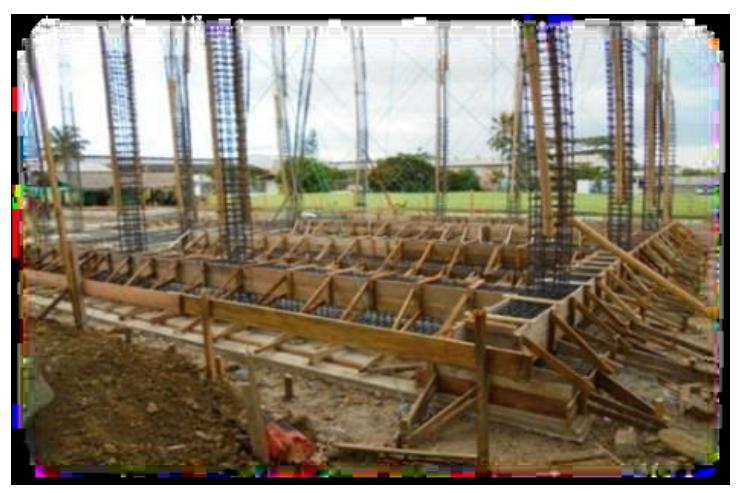

Para realizar el hormigón se usara concretera de un saco, para la cual se tomaran las medidas en parihuela o cajo netas. Dosificación en parihuelas para la preparación de hormigón en $1 \mathrm{~m}^{3}$.

- $\mathrm{F}^{\prime} \mathrm{c} 280 \mathrm{~kg} / \mathrm{cm}^{3}$

- 1 saco de cemento $50 \mathrm{~kg}$

- Arena 2 parihuelas de $15 \mathrm{~cm} \times 40 \mathrm{~cm} \times 40 \mathrm{~cm}$

- Piedra 2 parihuelas de $20 \mathrm{~cm} \times 40 \mathrm{~cm} \times 40 \mathrm{~cm}$

- Agua 25 litros 


\section{Elaboración de un plan para construir la cimentación de un edificio de tres plantas}

Vol. 2, núm. 2., (2018)

Luisa María Vega Benavides; Christian Eduardo Almendáriz Rodríguez; Iván Arturo Navarro

Veliz; Danny Xavier Almendáriz Rodríguez

\section{Figura $N^{\circ}$ 17.- Hormigonado}

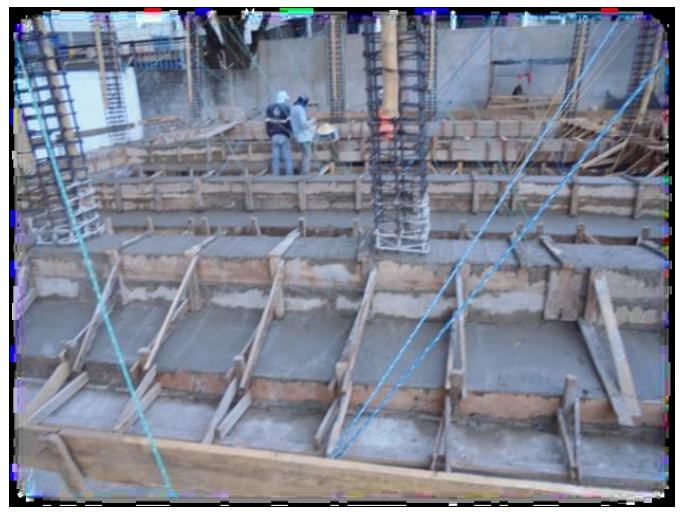

\section{Referencia: Fuente Propia}

El tambor de la concretera girara como mínimo 3minutos para poder iniciar con el hormigonado. Esta mezcla se le dará el uso inmediato luego del tiempo establecido ya que no se podrá mantener el concreto agregando más agua porque perderá su dosificación indicada y a su vez perderá resistencia. Este elemento debe utilizarse de 25 a 30 minutos máximo después de su mezcla, antes de su fraguado inicial. El concreto debe ser aplicado de manera correcta evitando que sus agregados se segreguen, esto quiere decir que se separen los agregados finos de los gruesos, lo ideal sería que el concreto trabaje de manera uniforme. Esta mezcla es transportada por medio de carretillas, que tomara un tiempo de 2 minutos de cargada del hormigón a la carretilla y 2 minutos de vaciado del hormigón, durante la ejecución del vaciado del hormigón se usara el vibrador para evitar huecos o vacíos en la estructura. El encofrado debe mantenerse húmedo al vaciar la mezcla para evitar la pérdida excesiva de líquido en el fraguado. El hormigón a usar tendrá las debidas especificaciones ya dadas. Antes del fraguado se realizara las pendientes laterales de la zapata. 


\section{Elaboración de un plan para construir la cimentación de un edificio de tres \\ plantas}

Vol. 2, núm. 2., (2018)

Luisa María Vega Benavides; Christian Eduardo Almendáriz Rodríguez; Iván Arturo Navarro Veliz; Danny Xavier Almendáriz Rodríguez

Al momento del hormigonado se procederá a tomar pruebas del hormigón en el sitio y en laboratorio.

- Cono de abrams.

- Rotura de cilindro de hormigón.

Figura $N^{\circ}$ 18.- Cono de abrams

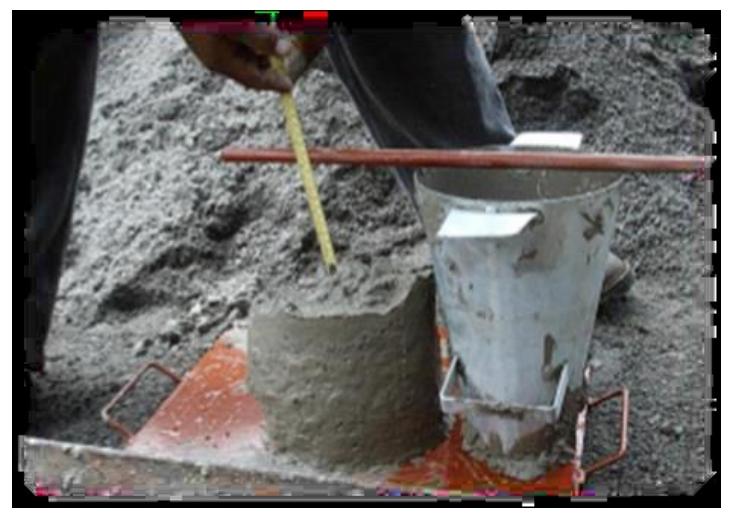

Referencia: Cimentación (U.N.A.M.)

Al término del hormigonado la estructura de cimentación, se debe iniciar con el curado el cual es indispensable para poder obtener la resistencia requerida ya que el hormigón no puede deshidratarse y este es muy necesario en los primeros 7 días que adquiere resistencia. Esta hidratación se realiza vertiendo agua sobre la estructura de cimiento.

Se procede a retirar el encofrado cuando el hormigón cumpla con un porcentaje mayor al $70 \%$ de la resistencia de diseño, esto se podrá efectuar en una duración de 12horas desde el momento de colocación del hormigón. 


\section{Elaboración de un plan para construir la cimentación de un edificio de tres plantas}

Vol. 2, núm. 2., (2018)

Luisa María Vega Benavides; Christian Eduardo Almendáriz Rodríguez; Iván Arturo Navarro Veliz; Danny Xavier Almendáriz Rodríguez

Hormigon ciclopeo $40 \%$ piedra $60 \%$ hormigón f'c $180 \mathrm{~kg} / \mathrm{cm}^{3}$.

Este tipo de hormigón se lo usan en los muros los cuales van colocados en diferentes partes de la estructura como es debajo de las riostras o muros sobre riostras.

Al emplear hormigón ciclópeo se debe respetar el porcentaje de piedra y hormigón el cual es $40 \%$ piedra y $60 \%$ hormigón. Consistirá en una mezcla de piedras tamaño entre 6 y 8 pulgadas.

Para iniciar el hormigonado se debe realizar el encofrado de los muros, utilizando tablas, cuartones y clavos. Las tablas se colocarán a lo largo del muro uniendo las tablas a lo alto de la dimensión del muro, por medio de cuartones y el uso de clavos. Estos cuartones se anclaran al suelo para que sirvan de estabilidad para el encofrado. En la parte superior del tablero se colocaran tiras de madera que van a estar clavadas a los tableros, las cuales van a evitar que los tableros se junten entre sí.

Figura $N^{\circ}$ 19.- Encofrado de Muros

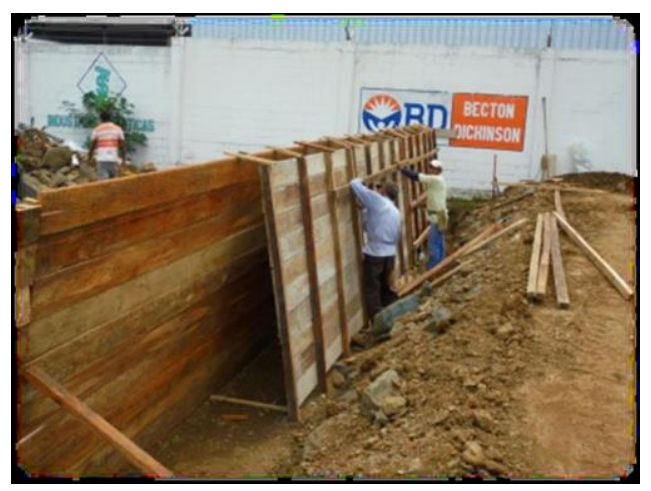

Referencia: Fuente Propia 


\section{Elaboración de un plan para construir la cimentación de un edificio de tres \\ plantas}

Vol. 2, núm. 2., (2018)

Luisa María Vega Benavides; Christian Eduardo Almendáriz Rodríguez; Iván Arturo Navarro Veliz; Danny Xavier Almendáriz Rodríguez

Al terminar la instalación del encofrado se colocan piedras de entra 6 y 8 pulgadas las cuales se las ubicara en toda la base del encofrado. Antes de iniciar el hormigonado se debe mantener húmeda el área donde se va a ubicar la mezcla de hormigón.

Dosificación en parihuelas para la preparación de hormigón en 1 m³.

- $\mathrm{F}^{\prime} \mathrm{c} 180 \mathrm{~kg} / \mathrm{cm}^{3}$

- 1 saco de cemento $50 \mathrm{~kg}$

- Arena 2 parihuelas de $15 \mathrm{~cm} \times 40 \mathrm{~cm} \times 40 \mathrm{~cm}$

- Piedra 4 parihuelas de $20 \mathrm{~cm}$ x $40 \mathrm{~cm}$ x $40 \mathrm{~cm}$

- Agua 170 litros

El tambor de la concretera girara como mínimo 3minutos para poder iniciar con el hormigonado. Esta mezcla se le dará el uso inmediato luego del tiempo establecido ya que no se podrá mantener el concreto agregando más agua porque perderá su dosificación indicada y a su vez perderá resistencia. Este elemento debe utilizarse de 25 a 30 minutos máximo después de su mezcla, antes de su fraguado inicial. El concreto debe ser aplicado de manera correcta evitando que sus agregados se segreguen, esto quiere decir que se separen los agregados finos de los gruesos, lo ideal sería que el concreto trabaje de manera uniforme. Esta mezcla es transportada por medio de carretillas, que tomara un tiempo de 2 minutos de cargada del hormigón a la carretilla y 2minutos de vaciado del hormigón, durante la ejecución del vaciado del hormigón se usara el vibrador para evitar huecos o vacíos en la estructura. El encofrado debe mantenerse húmedo al vaciar la mezcla para evitar la pérdida excesiva de líquido en el fraguado. 


\section{Elaboración de un plan para construir la cimentación de un edificio de tres plantas}

Vol. 2, núm. 2., (2018)

Luisa María Vega Benavides; Christian Eduardo Almendáriz Rodríguez; Iván Arturo Navarro Veliz; Danny Xavier Almendáriz Rodríguez

Al término del hormigonado los muros, se debe iniciar con el curado el cual es indispensable para poder obtener la resistencia requerida ya que el hormigón no debe deshidratarse y este es muy necesario en los primeros 7 días que adquiere resistencia. Esta hidratación se realiza vertiendo agua sobre la estructura de cimiento.

Se procede a retirar el encofrado cuando el hormigón cumpla con un porcentaje mayor al $70 \%$ de la resistencia de diseño, esto se podrá efectuar en una duración de 12horas desde el momento de colocación del hormigón.

\section{Conclusiones.}

Elaborar un plan para ejecutar un proyecto es de gran ayuda a la hora de llevar un control y administración; ya que nos permite realizar actividades ordenadamente, las cuales ya tendrán asignado su tiempo de ejecución esto quiere decir que cada actividad contara con tiempo de inicio y fin.

Para lo cual se tuvo que realizar un análisis minucioso de precios unitarios tomando en consideración mano de obra -cuadrilla-, maquinaria y materiales a utilizar. Para esto se consideró también la experiencia en campo de ingenieros, sobre el rendimiento de las cuadrillas según el rubro a utilizar. Ya que como factor principal depende de los rendimientos, el precio unitario de cada rubro.

En base a los rendimientos se podrán ejecutar actividades dándole así tiempo de inicio y fin a cada actividad. 


\section{Elaboración de un plan para construir la cimentación de un edificio de tres \\ plantas}

Vol. 2, núm. 2., (2018)

Luisa María Vega Benavides; Christian Eduardo Almendáriz Rodríguez; Iván Arturo Navarro Veliz; Danny Xavier Almendáriz Rodríguez

Los trabajos a ejecutarse los veremos reflejados en un cronograma valorado, el cual nos indicara su inicio y fin por rubro en cantidad de días, que según los rendimientos, se prolongara su duración.

\section{Bibliografía.}

Associação Brasileira de Normas Técnicas. (2006). NBR 12655: Concreto de cimento PortlandPreparo, controle e recebimento - Procedimento . Rio de Janeiro: ABNT.

Britez, C., Gadea, J., \& Carvalho, M. (2016). Hormigón y Hormigonado de Cimentación con Hormigón Autocompactante y de Elevadas Prestaciones (70MPa). Revista de la Asociación Latinoamericana de Control de Calidad, Patología y Recuperación de la Construcción, 6(1), 28-40.

Rodolfo, A. (1991). Suelo-Cemento. México: Instituto Mexicano del Cemento y el Concreto.

Spenser, D. (2014). La cimentación de la Confederación de Trabajadores de México. Tzintzun. Revista de Estudios Históricos, 60(Jul), 248-279.

Toirac Corral, J. (2008). EL SUELO-CEMENTO COMO MATERIAL DE CONSTRUCCIÓN. Ciencia y Sociedad, 33(4), 520-571. 